American Journal of Applied Sciences 4 (9): 653-657, 2007

ISSN 1546-9239

(C) 2007 Science Publications

\title{
Physical Nonlinearity Under Cyclic Loading In Neutron Flow
}

\author{
${ }^{1}$ Atwa. D. Zeyad and ${ }^{2}$ Starovoitov. E. I \\ ${ }^{1}$ Deparment of physics, Applied Science University (Private), Amman-11931, Jordan \\ ${ }^{2}$ Belorussian State Transport University, 246653, Gomel, Belorussia
}

\begin{abstract}
The influence of neutron irradiation on the nonlinear elastoplastic deformation of the constructions components under cyclic force disturbance was investigated. The formula of irradiation reinforcement was suggested depending on the experimental data, and the theorem of variable loading was proved. The problem of metal-polymeric sandwich beam was solved numerically.
\end{abstract}

Keywords: Cyclic loading, Neutron flow, Boundary-value problem, Sandwich beam

\section{INTRODUCTION}

Radiation treatment of rigid bodies is known to produce numerous effects which result in the appearance of additional volume deformations, and changes in the elastic (especially plastic) properties of the substance. Therefore it is necessary to bring in appropriate corrections into posing and solving the boundary-value problems concerning single and cyclic loading of elastoplastic components of the constructions. The main factors are the irradiation reinforcement of the substance (increasing of the yield point) and the irradiation swelling (increasing of volume deformation). Below is an attempt to extend Moskvitin's theory of variable loading ${ }^{[1]}$ by using the aforementioned class of boundary-value problems.

\section{Test Procedure}

Volume deformation and irradiation reinforcement: Consider initially a homogeneous isotropic body, occupying half-space $z \geq 0$. If parallel beam of neutrons with identical average energy and intention $\varphi_{0}$ falls upon the border $(z=0)$ parallel to the axe $z$, then the intention of neutrons reaching the plain $z=$ const, will be ${ }^{[2,3]} \varphi(z)=\varphi_{0} e^{-\mu z}$. where $\mu$ is called the macroscopic effective section and defined as $1 / \mathrm{m}$, where $m$ is the mass.

If $\varphi_{0}$ is independent of time, the flow of neutrons that will pass through the section $z$ up to the instance $t$

$$
I(z)=I_{0} e^{-\mu z} .
$$

where $I_{0}=\varphi_{0} t$, which is the total flow of neutrons per unit area of the surface of the body.
In rough estimation we may consider, that the change of the volume of the substance is directly proportional to the flow $I(z)$, and consequently $\theta_{I}=B I(z)$, where $B$ is an experimental constant. In reactors $\varphi_{0}$ lies in the range $10^{17}-10^{18}$ neutrons $/\left(\mathrm{m}^{2} . \mathrm{s}\right)$, and then $I_{0}$ can reach values of $10^{23}-10^{27}$ neutron $/ \mathrm{m}^{2}$. Consequently, depending upon the energy of the neutrons and the properties of the irradiated substance, $B$ can have the value of $10^{-28}-10^{-24} \mathrm{~m}^{2} /$ neutron and $\theta_{I}=$ 0.1 .

The dependence of the elasticity modulus, yield and solidity points, and the whole tension diagram upon $I_{0}$ for different neutron energies were experimentally investigated after irradiating the samples in nuclear reactors. Experimental results show that -as usual- the elasticity modulus was slightly changed (it had increased by $1.5-5 \%$ relative to that of the nonirradiated sample). On the contrary, the yield and solidity points are very sensitive to irradiation especially the yield point.

For massive bodies with a flat boundary, the number of neutrons passing at the depth $z$ under this boundary is estimated by formula (1), that's why the yield point will vary along $z$. At the surface of the body $(\mathrm{z}=0)$ the influence of irradiation upon the plastic limit $\sigma_{s}$ was characterised by the formula of irradiation reinforcement ${ }^{[2]}$ :

$\sigma_{s}=\sigma_{s 0}\left[1+A\left(1-\exp \left(-\xi I_{0}\right)\right)^{1 / 2}\right]$

where $\sigma_{s 0}$ is the plastic limit of the non-irradiated substance, A and $\xi$ are substance constants taken from the experiment. At the depth $z$ the formula takes the form

Corresponding Author: $\quad$ Atwa. D. Zeyad, Department of physics, Applied Science University(Private), Amman-11931, Jordan 


$$
\sigma_{s}=\sigma_{s 0}\left[1+A(1-\exp (-\xi I))^{1 / 2}\right]
$$

where the value of neutron flow $I(z)$ is given by equation (1). Let's denote appropriate values of deformation as $\varepsilon_{s 0}, \varepsilon_{s} ; A, \xi$ - are substance constants, taken from experiment. Fig.1 shows the relation between the satisfaction and the flow. If for example, $A$ $=1,09, \quad \xi=9,73 \cdot 10^{-26} \mathrm{~m}^{2} /$ neutron for aluminium alloy, then Fig. 1 indicates the satisfaction versus flow with known experimental data, where the dark points indicate the experimental data, and the solid line indicates the estimation by using formula (2).

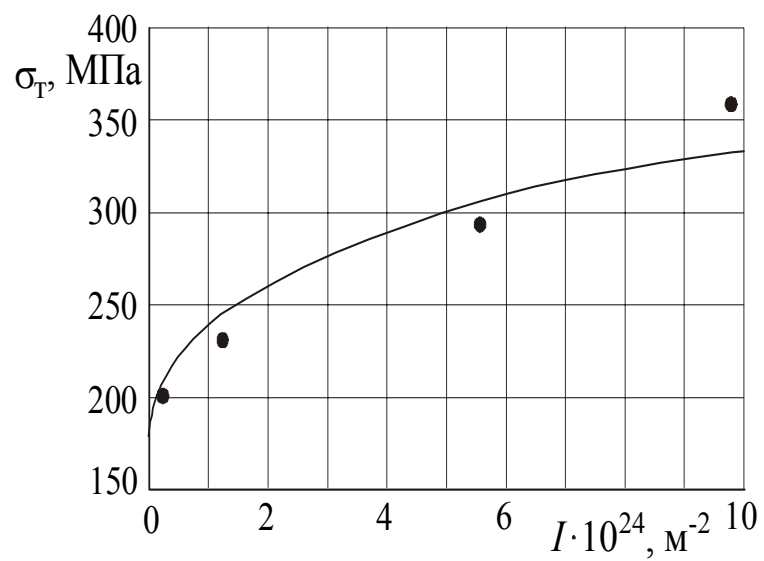

Fig 1: Satisfaction for aluminium alloy with $\mathrm{A}=1.09$ and $\zeta=9.73 \times 10^{-26}$. (Dark points represent the experimental data, and the solid line represents the estimation from equation 2.)

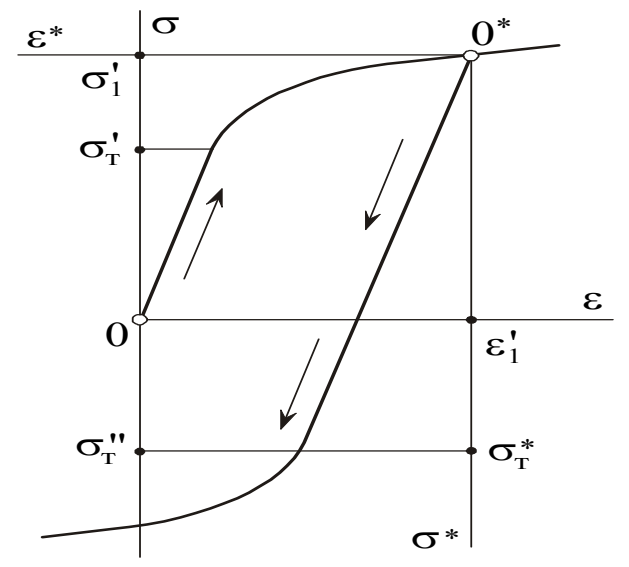

Fig. 2: The relation between elasticity point and deformation $\varepsilon$.

Statement of the problems of the theory of smallscale elastoplastic deformations: Consider the process of complex influence of external force and radiation loading upon a deformable body within the theory of small-scale elastoplastic deformations. The body which is in the natural state is supposed to be simultaneously influenced by instant external forces $F_{i}^{\prime}, R_{i}^{\prime}$ with a moving boundary of velocity $u_{i 0}$ ', and neutron flow $I_{0}=$ $\varphi_{0} t$. Under such influence it is assumed that areas of elastic and plastic deformations will appear in the body. Changes in the elasticity modulus caused by the neutron irradiation are neglected. Stresses, deformations and velocity of the body will be marked by one upper prime.

Hooke's law is valid in elastic areas of the solid body and the relations connecting the deviators of stress and deformation $s_{i j}^{\prime}, \ni_{i j}^{\prime}$ and also their spherical components $\sigma^{\prime}, \varepsilon^{\prime}$, are

$$
s_{i j}^{\prime}=2 G \ni_{i j}^{\prime}, \quad \sigma^{\prime}=K\left(3 \varepsilon^{\prime}-B I\right),
$$

where $B I$ is the correction due to additional volume deformation, caused by neutron irradiation, $G$ denotes the shear modulus, and $K$ the volumetric deformation modulus.

For those areas of the solid body, where plastic deformations had appeared the relation between the deviators for simple loading can be represented by

$$
s_{i j}^{\prime}=2 G \ni_{i j}^{\prime} f^{\prime}\left(\varepsilon_{u}^{\prime}, I, a_{k}^{\prime}\right) .
$$

where $f^{\prime}\left(\varepsilon_{u}^{\prime}, I, a_{k}^{\prime}\right)$ is the plasticity function which depends on the deformation intensity $\varepsilon_{u}{ }^{\prime}$, the value of neutron flow $I$ and the approximated parameters $a_{k}^{\prime}$. In the conditions of simple loading ${ }^{[1]}$ this function will be universal, i. e. it can be found from experiments with extension, torsion etc. So in the general case the relation between stresses and deformations in the deformable body under active loading from the natural state and under the influence of neutron flow can be represented by

$s_{i j}^{\prime}=2 G \ni_{i j}^{\prime} f^{\prime}\left(\varepsilon_{u}^{\prime}, I, a_{k}^{\prime}\right)$,

$\sigma^{\prime}=K\left(3 \varepsilon^{\prime}-B I\right)$.

In this case the plasticity function should be taken as $f^{\prime}\left(\varepsilon_{u}^{\prime}, I, a_{k}^{\prime}\right)=1$ in those areas, where $\varepsilon_{u}{ }^{\prime} \leq \varepsilon_{s}^{\prime}$, where $\varepsilon_{s}^{\prime}$ is the deformation corresponding to the plastic limit at the starting time.

If the force loading is rather quick (instantaneous), the irradiation reinforcement will not occur and the originated areas of plastic deformations will be the same as in the case of no influence of neutron flow. Though if the active loading will be slow enough, external layers of the body will turn out to be reinforced and within these layers areas of plastic deformations will turn out to be smaller or will be missed at all, compared with the non-irradiated body. There is an 
effect that can take place when the first plastic deformations appear not upon the external reinforced surface, but under it, where the deformation intensity is great and the plastic limit didn't increase. So the influence of irradiation upon elastoplastic bodies is opposite to the thermal inflounce, which decreases the plastic limit and results in increasing the areas of plastic deformations under equal loading.

Beside equation (3), Coshi relation and the following differential equations and the boundary conditions should be added on the assumption of infinitesimal deformations

$$
\begin{aligned}
& \sigma_{i j, j}^{\prime}+\rho F_{i}^{\prime}=0 ; \\
& \sigma_{i j}^{\prime} l_{j}=R_{i}^{\prime} \text { on } S_{\sigma}, \\
& u_{i}^{\prime}=u_{0 i}^{\prime} \text { on } S_{u} ; \\
& 2 \varepsilon_{i j}^{\prime}=u_{i, j}^{\prime}+u_{\mathrm{j}, i}^{\prime} .
\end{aligned}
$$

where the comma in the inferior index refers to the differentiation along next coordinate. Consider, the time variation of the external loading and the velocity of the boundary occur in such a way, that appropriate loading trajectories are not related to the class of essentially complex loadings, and the irradiation reinforcement takes place after force deformation of the solid body. Hereinafter we shall suppose that boundary value problem (3), (4) is solved.

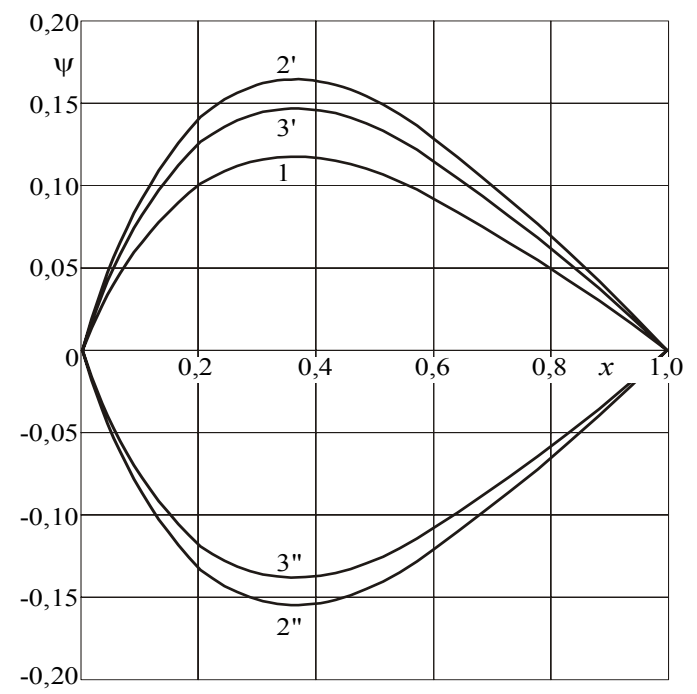

Fig. 3: The shear $\psi$ of the sandwich beam. The curves with one prime correspond to loading from natural state, while those with two primes represent the repeated cyclic winding due to alternating-sign loading: $l^{\prime}$ - solution of elastic problem: $2^{\prime} \quad$ - instant elastoplastic without irradiation; 3' elastoplastic winding of previously irradiated beam $\left(I_{1}=\right.$ $\left.5 \cdot 10^{24} \mathrm{M}^{-2}\right)$.
Problem definition for repeated alternating-sign loading: Suppose first that at $\mathrm{t}=\mathrm{t}_{1}$ there is no influence of neutron flow $(\varphi=0)$. Second, the external forces are changing so that in all points of plastically deformable areas of the body $V_{p}^{\prime}$ the unloading and following alternating-sign loading- by volumetric $F_{i}$ " and surface $R_{i}^{\prime \prime}$ forces (at $S_{\sigma}$ ) with boundary velocity $u_{0 i}$ " (at $S_{u}$ )take place. The level of irradiation of the body remains constant and equals its value before unloading $I_{1}=\varphi t_{1}$. The plastic limit in the points of the body depends upon the $z$ coordinate and becomes equal $\sigma_{s}{ }^{\prime \prime}\left(I_{1}(z)\right)$, i. e. it depends upon the value of the deformation and the irradiation reinforcement. The scheme of the process discussed is shown at fig. 2 .

Refer to the appropriate stresses, deformations and velocities as $\sigma_{i j}{ }^{\prime \prime}, \varepsilon_{i j}{ }^{\prime \prime}, \mathrm{u}_{i}{ }^{\prime \prime}$. Equation (4) is valid for these values

$$
\begin{aligned}
& \sigma_{i j}^{\prime \prime},{ }_{j}+\rho F_{i}^{\prime \prime}=0 ; \\
& \sigma_{i j}^{\prime \prime} l_{j}=R_{i}^{\prime \prime} \text { on } \\
& S_{\sigma}, \quad u_{i}^{\prime \prime}=u_{0 i}^{\prime \prime} \text { on } S_{u} ; \\
& 2 \varepsilon_{i j}^{\prime \prime}=u_{i}^{\prime \prime},{ }_{j}+u_{j}^{\prime \prime},_{i} .
\end{aligned}
$$

The relation between stresses and deformations will be

$$
s_{i j}^{\prime \prime}=2 G_{i j}^{\prime \prime} f^{\prime \prime}\left(\varepsilon_{u}^{\prime \prime}, \varepsilon_{1}^{\prime}, I_{1}, a_{k}^{\prime \prime}\right), \sigma^{\prime \prime}=3 K \varepsilon^{\prime \prime}
$$

Here $f^{\prime \prime}\left(\varepsilon_{u}^{\prime \prime}, \varepsilon_{1}^{\prime}, I_{1}, a_{k}^{\prime \prime}\right)$ is the plasticity function under repeated alternating-sign loading which depends upon the deformation intensity $\varepsilon_{u}{ }^{\prime \prime}$, the preceding values of deformation intensity $\varepsilon_{1}{ }^{\prime}$, the level of irradiation of the body $I_{1}$, and the approximated parameters $a_{k}{ }^{\prime \prime}$, describs the deformation curve of the second cycle.

At that point the plasticity function $f^{\prime \prime}$ is supposed to be equal to 1 in those areas where the new plastic deformations didn't appear, i. e. $\varepsilon_{u}{ }^{\prime \prime} \leq \varepsilon_{s}{ }^{\prime \prime}$, where $\varepsilon_{s}{ }^{\prime \prime}$ is the deformation that corresponds to the plastic limit $\sigma_{s}{ }^{\prime \prime}$ under repeated loading.

Equations (5), (6) define a boundary value problem for variables with double prime. The complexity of the problem arises from the dependence of the desired decision upon the unloading point $\left(\varepsilon_{1}{ }^{\prime}\right.$, $\sigma_{1}$ ) since the boundary value problem must be defined and solved at every point in the solid body. We will discuss one of the methods to avoid such difficulties.

For the values before the beginning of the unloading we shall retain designations $\sigma_{i j}{ }^{\prime}, \varepsilon_{i j}{ }^{\prime}, u_{i}{ }^{\prime}$. Following Moskvitin ${ }^{[1]}$ we shall define the following differences for times $t>t_{1}$ :

$s_{i j}^{*}=s_{i j}^{\prime}-s_{i j}^{\prime \prime}, \ni_{i j}^{*}=\ni_{i j}^{\prime}-\ni_{i j}^{\prime \prime}$. 
Consider the physical state equations for the variables with asterisks. The following relations are valid in the unloading and elastic deformation zones $V_{e}^{\prime}$ and $V_{e}^{\prime \prime}$;

$$
s_{i j}^{*}=2 G \ni_{i j}^{*}, \quad \varepsilon_{u}^{*} \leq \varepsilon_{\mathrm{T}}^{*}\left(I_{1}\right),
$$

the plastic deformation changes during variable loading in the area $\mathrm{V} p$ ", and the following relations must be valid

$s_{i j}^{*}=2 G \ni_{i j}^{*} f^{*}\left(\varepsilon_{u}^{*}, \varepsilon_{1}^{\prime}, I_{1}, a_{k}^{*}\right)$.

In general the function $f^{*}\left(\varepsilon_{u}^{*}, \varepsilon_{1}^{\prime}, I_{1}, a_{k}^{*}\right)$ appears as a new universal function depicting the nonlinearity of the deformation diagram $\sigma^{*} \sim \varepsilon^{*}$ (see fig. 2). On the linear section $f^{*}=0$.

The volumetric deformation remains elastic for all points of the body. Consequently before the beginning of the unloading and for current state, the following equalities are valid

$\sigma^{\prime}=K\left(3 \varepsilon^{\prime}-B I_{1}\right), \quad \sigma^{\prime \prime}=K\left(3 \varepsilon^{\prime \prime}-B I\right)$,

so that for values with asterisks,

$$
\sigma^{*}=3 K \varepsilon^{*}
$$

and the equilibrium equations, boundary conditions and Coshi relations for $\sigma_{i j}{ }^{*}, \varepsilon_{i j}{ }^{*}, u_{i}^{*}$ will be

$$
\begin{aligned}
& \sigma_{i j, j}^{*}+\rho F_{i}^{*}=0, \quad F_{i}^{*}=F_{i}^{\prime}-F_{i}^{\prime \prime} ; \\
& \sigma_{i j}^{*} l_{j}=R_{i}^{*}, \quad R_{i}^{*}=R_{i}^{\prime}-R_{i}^{\prime \prime}, \text { on } S_{\sigma} ; \\
& u_{i}^{*}=u_{0 i}^{*}=u_{0 i}^{\prime}-u_{0 i}^{\prime \prime}, \text { on } \\
& S_{u} ; \quad 2 \varepsilon_{i j}^{*}=u_{i}^{*},{ }_{j}+u_{j}^{*}{ }_{i} .
\end{aligned}
$$

Equations (8) - (10) make a new boundary value problem for the variables with asterisks. If we suppose that the function $f^{*}$ - in all points of the deformation curve- can be approximated by the function $f^{\prime}$, that is to describe it by the same analytical relation but with other parameters $a_{k}^{*}$ and exclude the dependence upon $\varepsilon_{1}$, then it will be given as:

$$
f^{*}=f^{\prime}\left(\varepsilon_{u}^{*}, I_{1}, a_{k}^{*}\right) \text {. }
$$

Comparing equations (3), and (4) for the body with loading from natural state and equations (8) -(10) with asterisks we find that they are very closely coincident within the approximations. Hence, the solution of the problem for the variables with asterisks can be obtained from knowing the solution of the problem of loading from the natural state by some replacements. For example, if the velocity $u_{i}^{\prime}=u_{i}^{\prime}\left(x, \varepsilon_{u}^{\prime}, \varepsilon_{s}^{\prime}, I, a_{k}^{\prime}\right)$ is known, then the velocity $u_{i}^{*}=u_{i}^{\prime}\left(x, \varepsilon_{u}^{*}, \varepsilon_{s}^{*}, I_{1}, a_{k}^{*}\right)$ and the velocity under repeated alternating-sign loading are calculated from relation (7) $u_{i}^{\prime \prime}=u_{i}^{\prime}-u_{i}^{*}$. Stresses and deformations are calculated by formulas of the same type.

\section{RESULTS}

Suppose that under $\mathrm{n}$-loading by external forces $F_{i}^{n}, R_{i}^{n}$ with boundary velocity $u_{0 i}^{n}$, the stresses $\sigma_{i j}^{n}$, deformations $\varepsilon_{i j}^{n}$ and velocity $u_{i}^{n}$ all appear. At the same time the equilibrium equations, boundary conditions and Coshi relations must be valid:

$\sigma_{i j, j}^{n}+\rho F_{i}^{n}=0 ; \quad \sigma_{i j}^{n} l_{j}=R_{i}^{n}$ on

$S_{\sigma}, u_{i}^{n}=u_{0 i}^{n}$ on $S_{u}$;

$2 \varepsilon_{i j}^{n}=u_{i}^{n},{ }_{j}+u_{j}^{n},{ }_{i}$.

Consider the differences:

$\sigma_{i j}^{* n}=(-1)^{n}\left(\sigma_{i j}^{n-1}-\sigma_{i j}^{n}\right), \quad \varepsilon_{i j}^{* n}=(-1)^{n}\left(\varepsilon_{i j}^{n-1}-\varepsilon_{i j}^{n}\right)$,

$u_{i}^{* n}=(-1)^{n}\left(u_{i}^{n-1}-u_{i}^{n}\right)$.

Then equations (11) turn out to be also valid for the varaibles with asterisks:

$\sigma_{i j}^{*_{n}{ }^{n},{ }_{j}}+\rho F_{i}^{*{ }^{*} n}=0, \quad F_{i}^{*_{n}}=(-1)^{n}\left(F_{i}^{n-1}-F_{i}^{n}\right) ;$

$\sigma_{i j}^{*_{n} n} l_{j}=R_{i}^{* n}$ on $S_{\sigma}, \quad u_{i}^{* n}=u_{0 i}^{* n}$ on $S_{u}$;

$R_{i}^{* n}=(-1)^{n}\left(R_{i}^{n-1}-R_{i}^{n}\right), \quad u_{0 i}^{* n}=(-1)^{n}\left(u_{0 i}^{n-1}-u_{0 i}^{n}\right)$;

$2 \varepsilon_{i j}^{*_{n}}=u_{i}^{*_{n},{ }_{j}}+u_{j}^{*_{n}},_{i}$.

Assume that under any $n$-loading the relation between spherical components of stresses and deformation tensors remains elastic. Repeating the previous supposition about possibility of curves $s_{i j}^{\prime} \sim \ni_{i j}^{\prime}$ and $s_{i j}^{*_{n}} \sim \ni_{i j}^{*_{n}}$ by nonlinear functions of identical analytic type

$s_{i j}^{*_{n} n}=2 G \ni_{i j}^{*_{n} n} f^{\prime}\left(\varepsilon_{u}^{*_{n}}, I_{1}, a_{k}^{*_{n}}\right)$,

we conclude that the solution of the problem for variables with asterisks (12), (13) under any given nloading may be obtained from the problem concerning the loading from the natural state. For example, if the velocity is known, $u_{i}^{\prime}=u_{i}^{\prime}\left(x, \varepsilon_{u}^{\prime}, \varepsilon_{\mathrm{T}}^{\prime}, I, a_{k}^{\prime}\right)$,

then the appropriate value with asterisk will be

$$
u_{i}^{*_{n}}=u_{i}^{\prime}\left(x, \varepsilon_{u}^{*_{n}}, \varepsilon_{\mathrm{T}}^{*_{n}}, I_{1}, a_{k}^{*_{n}}\right) \text {. }
$$

After this the desired velocity $u_{i}^{n}$ can be calculated from the relation

$u_{i}^{n}=u_{i}^{\prime}-\sum_{k=2}^{n}(-1)^{k} u_{i}^{* k}$ 
Stresses and deformations are calculated by formulas of the same type as (14).

As an example the problem of cyclic irradiationforce is winding of sandwich beam with one embed end. Asymmetry with respect to the thickness of the sandwich beam- with external bearing layers made of metal and incompressible with respect to the thickness of the internal layer (filler) made of polymer- is considered. For the description of the pack kinematics, the hypothesis of broken normal line is accepted: in the bearing layers Kirhgoff hypothesis is valid, and in the filler the normal line remains rectilinear without changing the length, but it turns on some additional angle $\psi(x)$. Bearing layers are accepted to be elastoplastic, and the filler is elastic. Analytical solution of the appropriate problem of the theory of elasticity is considered $\mathrm{in}^{[3]}$. Solving the problem of small elastoplastic deformations under loading from natural state was found by the method of elastic decisions. Aluminium alloy was used as a bearing layer, and Teflon was used as filler.

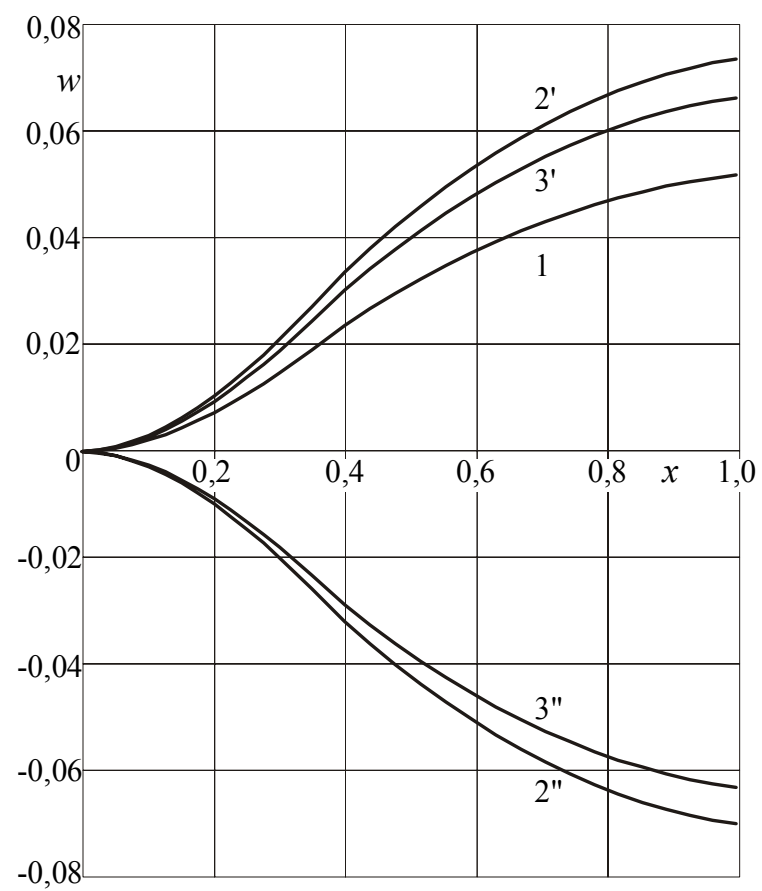

Fig. 4: The flexure $w$ of the sandwich beam. The curves with one prime correspond to loading from natural state, while those with two primes represent the repeated cyclic winding due to alternating-sign loading: $l^{\prime}$ - solution of elastic problem: $2^{\prime}$ - instant elastoplastic without irradiation; 3' elastoplastic winding of previously irradiated beam $\left(I_{1}=\right.$ $\left.5 \cdot 10^{24} \mathrm{M}^{-2}\right)$.
Appropriate mechanical properties of the media are considered $\mathrm{in}^{[2]}$. Figs. 3, 4 show the shear $\psi$ and flexure $w$ of the sandwich beam, which are calculated with the use of different physical state equations.

Under combined influence of force loading and neutron flow during interval $t_{1}$ till the value $I_{1}$ deformation will correspond to the curve $2^{\prime}$. Consequent instant unloading and force of alternatingsign loading with level of irradiation $I_{1}$ will cause the shear and flexure of the beam, shown by curves $2 "$. If the beam under cyclic loading was irradiated beforehand, then deformation would correspond to the curve $3 "$.

\section{CONCLUSION}

The theorem of cyclic loadings of elastoplastic bodies in neutron flow allows to simplify the whole class of boundary value problems. However, it is necessary to point out to the limits of its application. First, maximum level of neutron irradiation must not cause the loosening of the matter. Second, at every halfcycle the conditions of simple loading must be fulfilled and deformations must be small.

\section{ACKNOWLEDGEMENT}

The financial support from the Applied Science University is gratefully acknowledged.

\section{REFERENCES}

1. Moskvitin V.V., 1981. Cyclic loadings of construction elements: Russia, Moscow Nauka Publishers, pp : 344-367

2. Starovoytov E.I., 2000. The basics of the theory of elasticity, plasticity and viscoelasticity: Russia, Gomel: Belaruskaya Nauka Publishers, pp: 330345

3. Starovoytov E.I., 2002. Viscoelastoplastic layer plates end shells: Gomel: Belaruskaya Nauka Publishers , pp: 343-358

4. Starovoytov E.I., Yarovaya A. V., 1988. Viscoelastoplastic beam under thermo force loadings: Mechanics of solid body, № 3: 109116 